\title{
Analysis of Distributed Traffic Control Schemes in Web Attacks
}

\author{
M. Paranthaman ${ }^{1}$, Agnus S. Swarna Nishalakshmi ${ }^{2}$ \\ Assistant Professor, Department of ECE, M. Kumarasamy College of Engineering, Karur, India ${ }^{1,2}$
}

\begin{abstract}
A novel distributed traffic control service is used, which will help us to improve Internet security significantly. At its core is a safe delegation of network management capabilities. It is based on adaptive network traffic processing devices that can be deployed incrementally in the Internet close to routers. Our service can help to stop attack traffic within the network as close to the internet uplink of an attacker as possible. The common aim of DDoS attacks is to deny certain services or resources to prospective users. The mitigation schemes even strengthen the belongings of an attack as genuine servers or widespread networks are cut off from the network.
\end{abstract}

Keywords: DDoS attacks, Digital Signature, Network management, Traffic control.

\section{INTRODUCTION}

The Internet was meant to deliver a communications network that might work though a number of the foremost sites were down. If the shortest route wasn't on the market, routers would straight traffic round the network via totally different routes [4]. The net at the beginning was employed by laptop specialists, engineers, scientists, and librarians. There was nothing hospitable concerning it. There have been no home or workplace personal computers in those days, and anyone World Health Organization used it, whether or not a laptop skilled or associate degree engineer or human or professional, had to be told to use an awfully complicated system [9]. As the web has become present, faster, and more and more accessible to non-technical communities, social networking and cooperative services have adult quickly, sanctioning individuals to speak and share interests in more ways in which. Sites like Facebook, Twitter, LinkedIn, YouTube, Flickr, Second Life, delicious, blogs, wikis, and lots of a lot of let individuals of all ages quickly share their interests of the instant with others everyplace [2]. Network security consists of the provisions and policies adopted by a network administrator to forestall and monitor unauthorized access, misuse, modification, or denial of a laptop and network-accessible resources.

Network security involves the authorization of access to knowledge in an exceedingly network, that is controlled by the network administrator [15]. Users opt for are allotted associate ID and password or alternative authenticating data that permits them access to data and programs at intervals their authority. Network security covers a spread of pc networks, each public and personal, that area unit utilized in errands conducting transactions and communications among businesses, government agencies and people [1]. Networks are non-public, like at intervals an organization, et al which could be hospitable public access. Network security is concerned in organizations, enterprises, and alternative varieties of establishments. Network security starts with authenticating the user, unremarkably with a username and a watchword. With two-factor authentication, one thing the user 'has' is additionally used (e.g. a security or 'dongle', associate ATM card, or a mobile phone); and with three-factor authentication, one thing the user 'is' is additionally used [6]. Once attested, a firewall enforces access policies like what services area unit allowed to be accessed by the network users though effective to stop unauthorized access, this part could fail to ascertain doubtless harmful content like pc worms or Trojans being transmitted over the network. Anti-virus software package or associate intrusion interference system (IPS) facilitate sight and inhibit the action of such malware [5]. Associate anomalybased intrusion detection system might also monitor the network and traffic for sudden (i.e. suspicious) content or behavior and alternative anomalies to guard resources, e.g. from denial of service attacks or associate worker accessing files at strange times. Individual events occurring on the network could also be logged for audit functions and for later high-level analysis [3]. Communication between two hosts employing a network could also be encrypted to take care of privacy. Honey pots, primarily decoy network-accessible resources, could also be deployed in an exceedingly network as police work and early-warning tools, and because the honey pots don't seem to be usually accessed for legitimate functions [7]. Techniques utilized by the attackers that conceive to compromise these decoy resources area unit studied throughout associated when an attack to stay a watch on new exploitation techniques. Such analysis could also be accustomed any tighten security of the particular network being protected by the honeypot [8].

\section{DDOS ATTACKS}

A distributed denial of service attack (DDoS) happens once multiple systems flood the information measure or resources of a targeted system, sometimes one or a lot of 
Vol. 6, Issue 1, January 2017

internet servers [11]. These systems are compromised by tools area unit supposed for benign use, however they'll attackers employing a style of ways. A system may even be used in launching attacks on victim networks [21]. additionally be compromised with a trojan, permitting the aggressor to transfer a zombie agent. Attackers may also burgled systems victimization machine-controlled tools that exploit flaws in programs that listen for connections from remote hosts. This state of affairs primarily issues systems acting as servers on the online. Distributed denialof-service (DDoS) attacks are a real-and growing-threat to businesses worldwide [16]. Designed to elude detection by today's hottest tools, these attacks will quickly incapacitate a targeted business, cost accounting victims thousands, if not millions, of bucks in lost revenue and productivity. By adopting new purpose-made solutions designed specifically to find and defeat DDoS attacks, businesses will keep their business operations running swimmingly. If associate assaulter mounts associate attack from one host it might be classified as a DoS attack. In fact, any attack against convenience would be classed as a Denial of Service attack [10]. On the opposite hand, if associate assaulter uses several systems to at the same time launch attacks against a distant host, this is able to be classified as a DDoS attack. DDoS traffic with time to measure (TTL) data at the routers by applying the support vector machine (SVM) module to regulate malicious traffic and manage DDoS attack packets with efficiency. The DDOS tool is employed in several functions. It utilizes a stratified structure wherever the assaulter uses a shopper program to attach to handlers, that area unit compromised systems that issue commands to the zombie agents that successively facilitate the DDoS attack [12].

Agents are negotiated via the handlers by the assaulter, victimization machine-controlled routines to use vulnerabilities in programs that settle for remote connections running on the targeted remote hosts. Every handler will management up to $\mathrm{k}$ agents. the main blessings to associate assaulter of employing a distributed denial-of-service attack area unit that: multiple machines will generate additional attack traffic than one machine, multiple attack machines area unit tougher to show off than one attack machine, which the behavior of every attack machine will be stealthier, creating it tougher to trace and finish off [14]. These assaulter blessings cause challenges for defense mechanisms. a good array of programs is employed to launch DoS-attacks. Most of those programs area unit fully centered on playacting DoS-attacks, whereas others are true Packet injectors, therefore able to perform different tasks additionally. Such

DDoS attacks a very severe hazard to computers users. A Distributed Denial of Service (DDoS) Attack is collected of four elements

- The real attacker.

- The handlers

- The attack daemon agents or zombie hosts

- A victim or target host.

Here, we have to introduce four types of concepts. They are Digital Signature, Trace Back, Network Monitoring and also the K-NN classification. The following steps take place while preparing and conducting a DDoS attack [8]

- Assortment of agents

- Conciliation

- Communication

- Outbreak

\section{SYSTEM ANALYSIS}

In our suggested system we have presented the trace back method. The method is centered on packet marking approach to evade storing state at routers. As an alternative of inserting its entire IP address into the packet, each node inserts only the part of the IP address to specify its occurrence on the path. This method provisions the network forensics by sampling the hints of distrustful network activity [22]. Beforehand forwarding a packet the router inserts the IP address of its output interface into the packet. Herein router inserts its outer-interface IP address into the forward packet. Upon getting an attack packet, the target positions whose elements are the routers that compose the attack path. To reconstruct the attack path, the following procedure is used. Primarily the target authorizes for the occurrence of all neighbor routers in the received attack networks. The network standings are attack, pre-attack and normal [17].

- The Light-Weight Hash function is utilized here to confirm the digital signature of the particular clients. This is mostly used to preserve the security of the system performance [18].

- The k-NN algorithm is a similarity-based learning algorithm and is known to be highly active in several problem domains, counting classification problems [13].

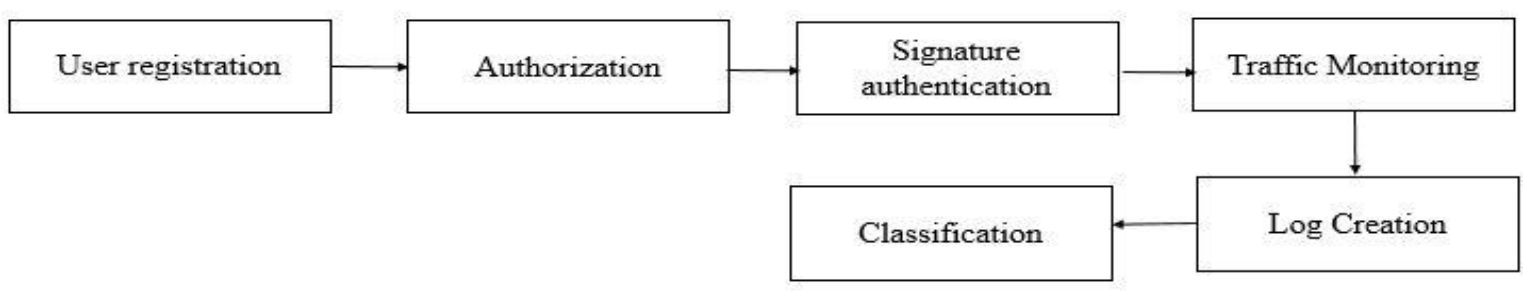

Figure 1. System Architecture 
Vol. 6, Issue 1, January 2017

- The k-NN method is used because this method has features that are appropriate for our goals. These features are: easy execution, short time computation, and high precision [23].

The test element has to be given and the k-NN algorithm finds its $\mathrm{k}$ nearest neighbors among the training elements, which form the neighborhood. The algorithm based on the cosine formula is most prevalent method used for approximating the similarity degree [19].

- We train three datasets - normal, pre-attack, and attack datasets [23]. Every part in each dataset has nine elements that are computed from the data log for the period.

- We calculate the current network status as an element with nine components in that period and apply the distance formula to find the $\mathrm{k}$ nearest neighbors of the current network status [20].

\section{SYSTEM DESIGN}

In first the client has to register the valid details. After the registration process over the digital signature has been generated and transferred to the client mail. Then the client has to enter the valid login details at each time. If the user has not given the valid login details not enter into the process [21]. After enter the login details the server has to verify the signature of the client. If the signature of the client is true the client easily enters into the process. If the signature of the client is false the particular user is an attacker. The entire process is explained in Fig. 1 that the traffic has not occurred with status normal, source and destination IP address.

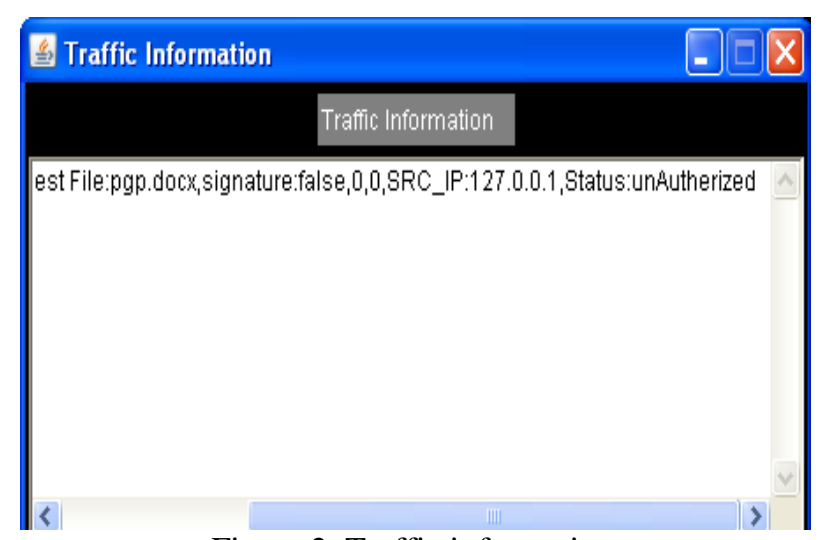

Figure 2. Traffic information

In our classification module we have to notice the status of the network. The network statuses are normal, pre-attack and attack. This method uses two processes. One is offline and another one is on-line. After the trace back method was over the classification process will be included. The attack has to be finding the particular process can be blocked. The training data should be viewed and check the status of the data's accordingly. Already stored status about the network can be classified.

\section{RESULTS AND DISCUSSION}

The signature is a process of demonstrating the authenticity of a digital message. A valid digital signature gives a recipient reason to believe that the message was created by a known sender, and that it was not altered in transit. Digital signatures are commonly used for software distribution, financial transactions, and in other cases where it is important to detect forgery or tampering. The signature of the client file will be writing into the server side only. By sending the IP-address and port number of the client immediately the server can check the authenticity of the particular client. The figure 2 indicates Figure 3 indicates that the registration and log in process of the desired user. The attack is denoted as unauthorized in traffic information shown in Fig.3.

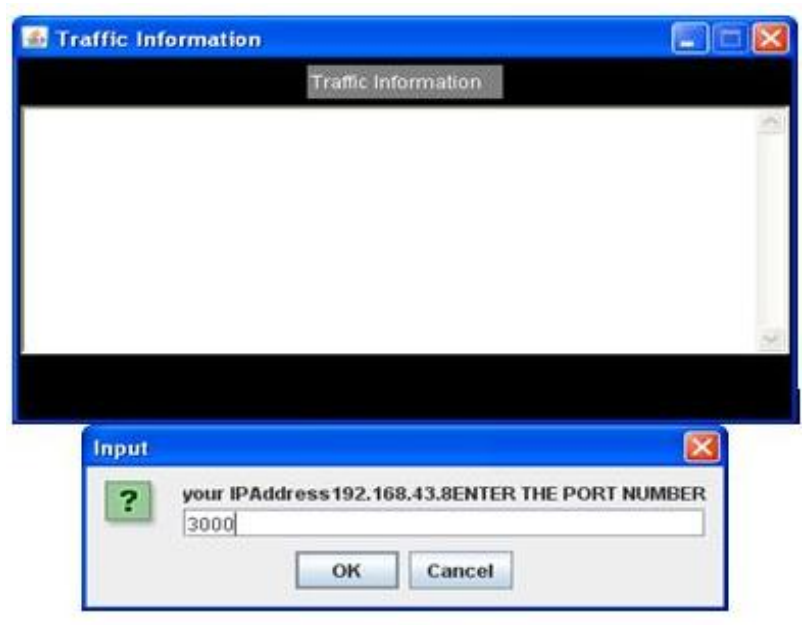

Figure 3. Log in Creation

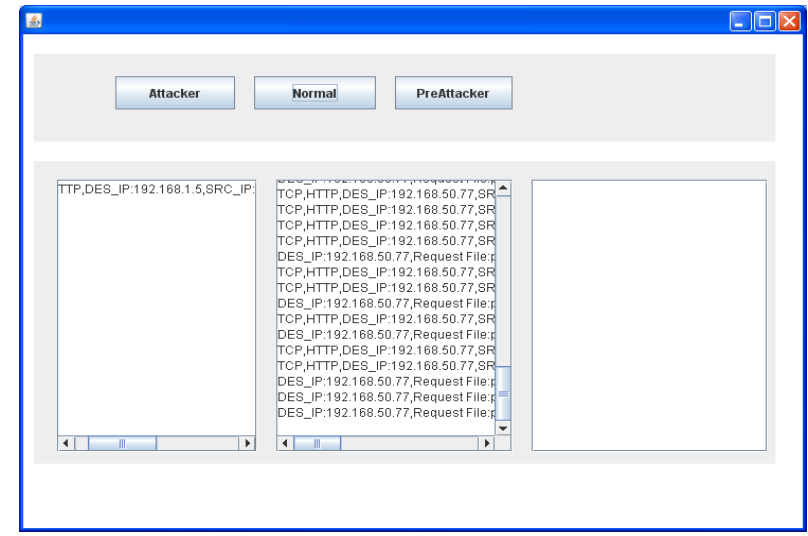

Figure 4. Classification output

\section{CONCLUSION}

We have proposed a simple web based traffic control system that has given the freedom to establish diverse applications within the network and to securely provider the partial network control to network users. We have worked out a separate filtering for malevolent traffic through operative trace back technique to diminish DDOS attacks engendered. Data mining has become easier 
Vol. 6, Issue 1, January 2017

through our refined approach and our classifier provides good accuracy in identifying normal, attacker and pre attacker.

\section{REFERENCES}

[1] Ambrose P J, Rai A, Ramprasad, "Internet usage for information provisioning: theoretical construct development and empirical validation in the clinical decision making context", IEEE transaction on engineering, pp :661-667, 2006

[2] R.Dhivya, V.Kavitha, "Secured Client Cache Sustain for Maintaining Consistency in MANET's", International Journal of Research in Engineering and Technology, Vol. 3, Issue 7, pp.1-6, 2014

[3] D"ubendorfer, Matthias Bossardt, Bernhard Plattner,"Adaptive Distributed Traffic Control Service for DDoS Attack Mitigation", 19th IEEE International Parallel and Distributed Processing Symposium, 2006

[4] V.Kavitha, C.Gayathri, "A Survey on Detection Methods for Network Layer Attacks in WMN's", International Journal of Applied Engineering Research, Vol.10, Issue 1, pp.744-748, 2015.

[5] S.Palanivel Rajan, R.Sukanesh, "Experimental Studies on Intelligent, Wearable and Automated Wireless Mobile Tele-Alert System for Continuous Cardiac Surveillance", Journal of Applied Research and Technology, ISSN No.: 1665-6423, Vol. No. 11, Issue No.: 1, pp.133-143, 2013.

[6] V.Kavitha, S.V.Vidya, "A grid based vehicle localization system providing safety precautions", International Journal of Applied Engineering Research, Vol.10, Issue 1, pp.940-944, 2015.

[7] Jean-Philippe Aumasson, Luca Henzen, Willi Meier, and Maria Naya-Plasencia.“ Quark: A lightweight hash", Mangard and Standaert, 2012.

[8] S.Vijayprasath, S.Palanivel Rajan, "Performance Investigation of an Implicit Instrumentation Tool for Deadened Patients Using Common Eye Developments as a Paradigm", International Journal of Applied Engineering Research, ISSN No.: 0973-4562, Vol. 10, Issue No.1, pp. 925-929, 2015.

[9] R.Renuka, V.Kavitha, "A Performance Analysis of Load Balanced Deflection Routing with Priority Scheduling in OBS Networks", International Journal of Engineering, Science and Technology, Vol. 5, Issue 4, pp.7-13, 2013.

[10] S.Palanivel Rajan, R.Sukanesh, S.Vijayprasath, "Analysis and Effective Implementation of Mobile Based Tele-Alert System for Enhancing Remote Health-Care Scenario", HealthMED Journal, ISSN No. : 1840-2291, Vol. No. 6, Issue 7, pp. 2370-2377, 2012.

[11] K. Park and W. Willinger,"Self-similar network traffic and performance evaluation", Wiley New York, 2000.

[12] S.Palanivel Rajan, R.Sukanesh, S.Vijayprasath, "Design and Development of Mobile Based Smart Tele-Health Care System for Remote Patients", European Journal of Scientific Research, ISSN No.: 1450-216X/1450-202X, Vol. No. 70, Issue 1, pp. 148-158, 2012.

[13] Kavitha, V., and Palanisamy, V. "New Burst Assembly And Scheduling Technique For Optical Burst Switching Networks", Journal of Computer Science, 9, (8), pp. 1030,2013.

[14] S.Palanivel Rajan, Sukanesh, R, et.al., "Performance Analysis Of Mobile Phone Radiation Minimization Through Characteristic Impedance Measurement", International Journal of Computer Science Issues, Vol. 9, Issue 2, p540, 2012.

[15] Deepa, E., Palaniswamy, R., \& Kuppusamy, S., Effect of performance appraisal system in organizational commitment, job satisfaction and productivity. Journal Of Contemporary Management Research, 8(1), 72-82, 2014.

[16] S.Palanivel Rajan, K.Sheik Davood, "Performance Evaluation on Automatic Follicles Detection in the Ovary", International Journal of Applied Engineering Research, ISSN No.: 0973- 4562, Vol. 10, Issue 55, pp. 1-5, 2015.

[17] Mohanpriya S., Vadivel M, "Automatic Retrieval of MRI Brain Image using Multiqueries System”, IEEE Conference, pp 10991103, 2013.

[18] S.Palanivel Rajan, "Review and Investigations on Future Research Directions of Mobile Based Telecare System for Cardiac
Surveillance", Journal of Applied Research and Technology, ISSN No.: 1665-6423, Vol. 13, Issue 4, pp. 454- 460, 2015.

[19] Andrey Bogdanov, Miroslav Knezevic, Gregor Leander, Deniz Toz, Kerem Varici, and Ingrid Verbauwhede, "SPONGENT: A lightweight hash function", CHES, volume 6917 of LNCS, pages 312-325. Springer,2011

[20] Dr.C.Vivek, S.Palanivel Rajan, "Z-TCAM : An Efficient Memory Architecture Based TCAM", Asian Journal of Information Technology, ISSN No.: 1682-3915, Vol. No.: 15, Issue : 3, pp. 448454, 2016.

[21] Kavitha, V. and V. Palanisamy, "Simultaneous Multi-Path Transmission for Burst Loss Recovery in Optical Burst Switching (OBS) Networks," Eur. J. Sci. Res., 87: pp. 412-426, 2012.

[22] S.Palanivel Rajan, T.Dinesh, "Analysis of Human Brain Disorders for Effectual Hippocampus Surveillance", International Journal of Modern Sciences and Engineering Technology, ISSN 2349-3755, Vol. No.: 2, Issue No.: 2, pages: 38-45, 2015.

[23] S.Palanivel Rajan, M.Paranthaman, Dr.C.Vivek, (2016) "Design and Enhancement of Wideband Reconfigurability using Two EShaped Patch Antenna", Asian Journal of Research in Social Sciences and Humanities, ISSN : 2249-7315, Vol.6, Issue 9, pp. 317-327

[24] S.Palanivel Rajan, "Diagnosis of Cardiovascular Diseases using Retinal Images through Vessel Segmentation Graph", Current Medical Imaging Reviews, Online ISSN: 1875-6603, ISSN: 15734056, Vol.: 13, DOI: 10.2174/15734056136661701111 53207, 2017.

[25] M. Chandrasekar, S. Rajkumar, D. Valavan, A review on the thermal regulation techniques for non integrated flat PV modules mounted on building top, Energy Build. 86, 692-697, 2015.

[26] S.Palanivel Rajan, T.Dinesh, "Systematic Review on Wearable Driver Vigilance System with Future Research Directions", International Journal of Applied Engineering Research, ISSN No.: 0973-4562, Vol. 10, Issue No.1, pp. 627- 632, 2015.

[27] S.Palanivel Rajan, "A Significant and Vital Glance on "Stress and Fitness Monitoring Embedded on a Modern Telematics Platform", Telemedicine and e-Health Journal, ISSN: 1530- 5627 (Online ISSN: 1556-3669), Vol. No.: 20, Issue No.: 8, pages: 757-758, 2014. 